\title{
New Sky Pattern Recognition Algorithm
}

\author{
Wojciech Makowiecki ${ }^{1}$ and Witold Alda ${ }^{2}$ \\ ${ }^{1}$ Astronomical Observatory of the Jagiellonian University, Kraków, Poland \\ wojciech.makowiecki@gmail.com \\ http://www. wojciech.us \\ ${ }^{2}$ AGH University of Science and Technology, Kraków, Poland \\ alda@agh.edu.pl
}

\begin{abstract}
We present here a new algorithm which enables the identification of stars in astronomical photographs using readily available star catalogs for this purpose. The algorithm was implemented in a standalone application called 'Skyprint' capable of performing a matching process. The computational aspect of the problem can be designated to the wide class of image recognition methods and analysis of multidimensional data. The astronomical aspect concentrates on astrometry the method of determining the coordinates of stars in the celestial sphere. The problem of identifying star patterns occurs most often in such areas as cosmic probe navigation, adjusting and merging numerous photographs of the sky together, or in recovering missing information in relation to a fragment of the sky represented in the photograph.
\end{abstract}

Keywords: Pattern recognition, Astrometry, Geometric hashing.

\section{Introduction}

The concept of matching the stars in a photograph with stars in the catalog has been investigated for many years. The majority of algorithms which are able to successfully solve the problem are based on the idea of comparing polygonal shapes in the photograph with those in the catalog. The shapes in the photograph are created by connecting stars in the photograph together and likewise the shapes in the catalog.

We can distinguish two strategies of approaching the problem of matching these two groups of shapes. The first one, used for example by Valdes [1], Groth [2] and Murtagh [3], relies on heavy restrictions that are put on the fragment of the catalog to be compared with the photograph. We must know that the chosen fragment of the catalog contains most of the stars visible in the photograph. Also the size of the fragment can not be larger than a few times the size of the field of view (FOV) shown on the photograph. The shapes (in this case triangles), made up of stars brighter than a certain limiting magnitude, are created in the picture and in the chosen part of the catalog. Then the triangles are saved in two separate lists so that they can be later investigated for similarities. Confirmation that two found shapes are made up of the same objects is done in the so called 'voting process'.

M. Bubak et al. (Eds.): ICCS 2008, Part I, LNCS 5101, pp. 749 758, 2008.

(C) Springer-Verlag Berlin Heidelberg 2008 
In this paper the authors have concentrated on proposing a new algorithm belonging to the second group, that has emerged recently e.g. Lang [4.

Algorithms belonging to this group are able to recognize an arbitrary fragment of the catalog shown on the photograph, without being limited by cases where the approximate location is not known. Such algorithms are not usually able to identify particular stars belonging to the shape, however, for this purpose, one of the algorithms from the first group which has already been developed, can be chosen. The main idea behind the algorithm presented is to create and subsequently compare two sets of convex quads. The first set is created by using the stars found in the photograph, and the second one by using the stars present in the catalog.

\section{Algorithm}

One of the most important issues in the algorithm is its method of characterizing the shapes. We need to do this in a way which is invariant with respect to translation, rotation and scaling. Each shape consists of $N$ points. Each point has 2 coordinates, therefore we need $2 N$ parameters to determine the positions of all $N$ points in the 2-dimensional space. As translation, rotation and scaling in a $2 D$ coordinate system require 4 degrees of freedom, we end up with the $2 N-4$ parameters which determine the shape unambiguously. This gives us 2 parameters for each triangle and 4 parameters for each quad.

In our approach we have chosen to use quads and to compare them by using only 3 internal angles of each quad. Although such a measure is not sufficient to determine the convex quad unambiguously, we sacrifice this for the advantage of having only three parameters for each shape. Our tests show that such an approach performs well and is sufficient in most cases.

The algorithm consists of several steps:

\section{Creating the 'grid of seeds'}

By 'seed' we mean a virtual point having 2 coordinates (Right Ascension and Declination). We define 'grid of seeds' as points distributed in one of any number of regular ways. For example one can choose to satisfy the following condition:

$$
\frac{\text { number of seeds per unit area }}{\text { number of stars per unit area }} \approx \text { const. }
$$

We believe this rule of placing seeds is one of the best.

2. Choosing only the convex shapes for later comparison

Now we use the seeds to create shapes as shown in Fig. 1. We try to create a convex quad in the vicinity of each seed. A case with three collinear stars is treated as a correct convex quad.

\section{Angle calculation, discretization and hashing}

In this stage we first calculate angles by using one of three different methods mentioned later. Subsequently we discretize angles every 2 degrees. For each shape we store 5 values: 3 discrete angles and 2 coordinates of the seed which 
a)

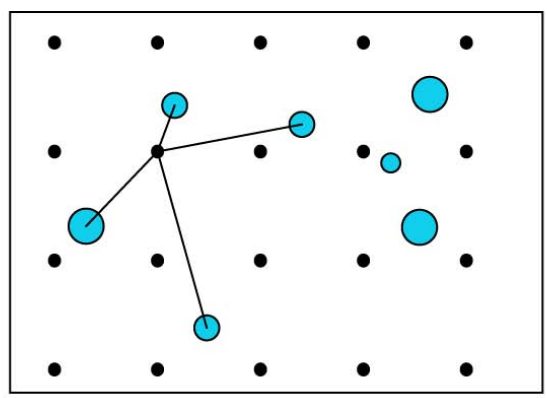

c)

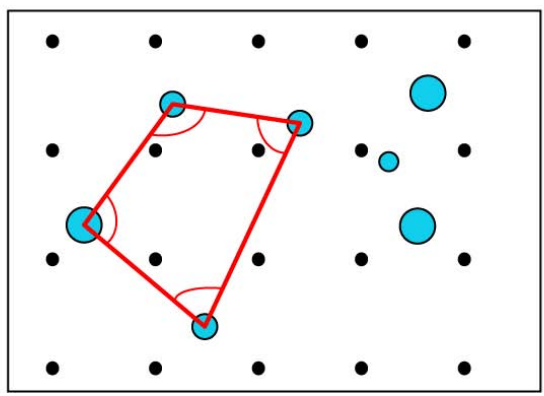

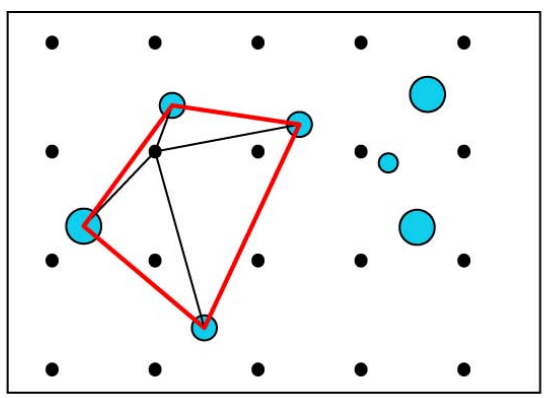

b)

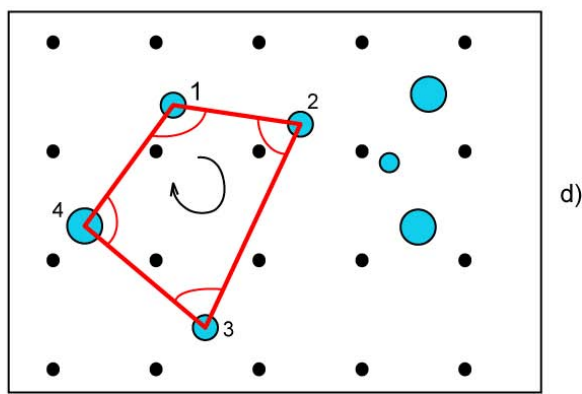

Fig. 1. Stages of creating a convex quad. Black dots denote seeds of the grid, blue circles denote stars. Sequence of stages are as follows:

a) select one seed and choose 4 closest stars

b) create quad only if these 4 stars can make a convex one

c) calculate internal angles of the quad

d) choose the largest angle and two more angles by moving in a clockwise direction, remember values and sequence of these 3 angles.

was used to create this particular quad. We use a standard hash function with primes as coefficients in the form:

$$
(\alpha * 23827+\beta * 34693+\gamma * 46021) \% 904997
$$

which transforms 3 input angles $(\alpha, \beta$ and $\gamma)$ to an array index. This method enables quick matching of quads.

4. Division of sky into fragments

In this step, in order to use "voting', we divide the whole sky into fragments as shown in Fig. 2, 3 and 4. Fragments can have different shapes and sizes so there are many different ways of doing this. In our approach we use orthogonal division. We limit the minimum size of the fragments to the maximum FOV of the input photograph. In other words, the minimum size of the fragment must be larger than the photograph. However, the limiting size cannot be too large - because in such a case, identifying fragments would be imprecise or incorrect. Even when the photograph has angular dimensions which fit in the single fragment of the catalog, it may be only partially contained. Figure 2 shows our solution to this problem. We enlarge the fragment 


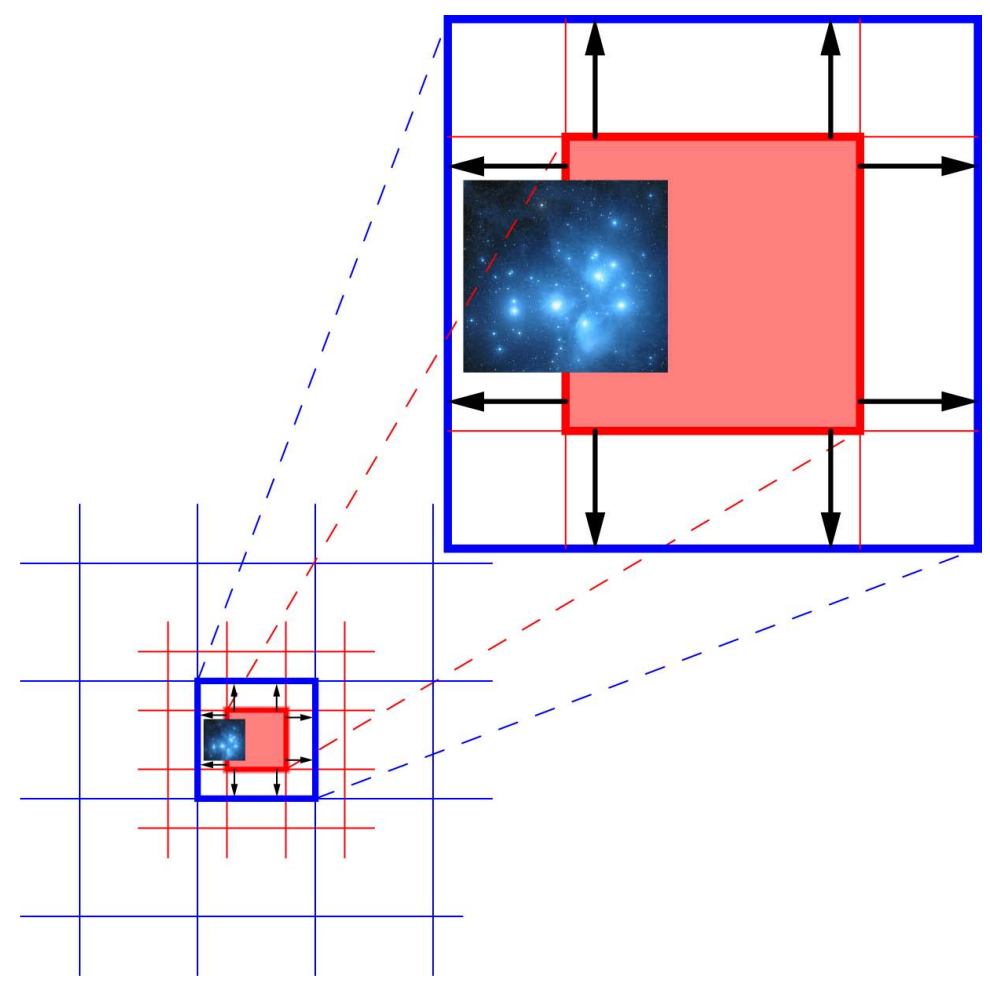

Fig. 2. Enlarging fragments of the catalog by a factor of 2 in order to be sure that the photograph is always entirely contained by at least one fragment

by a factor of 2 in order to be sure that the photograph is always entirely contained by at least one fragment.

\section{Counting matched quads and voting on catalog fragments}

We then match the convex quads from the photograph with those in the catalog. Once a match is found, one or more areas in the sky receives votes as shown in Fig. 3. At the end of the process, the region of the catalog which has received the highest number of votes is the one we were looking for (Fig. 4).

We use seeds for two reasons. Firstly, to introduce a unique and easy method for choosing stars, which will be used later to create quads. Secondly, using seeds is necessary for reducing the number of convex quads created from the catalog. The problem shows certain asymmetry. For the photograph, we can create as many convex quads as possible without using seeds at all. The number of convex quads is the main reason for this. A photograph always has many fewer stars than the whole sky catalog. However, as it turned out in our tests, such a method is not optimal and introduces difficulties. The main problem concerns the scale of quads. If we create all possible convex quads from the stars in the photograph 


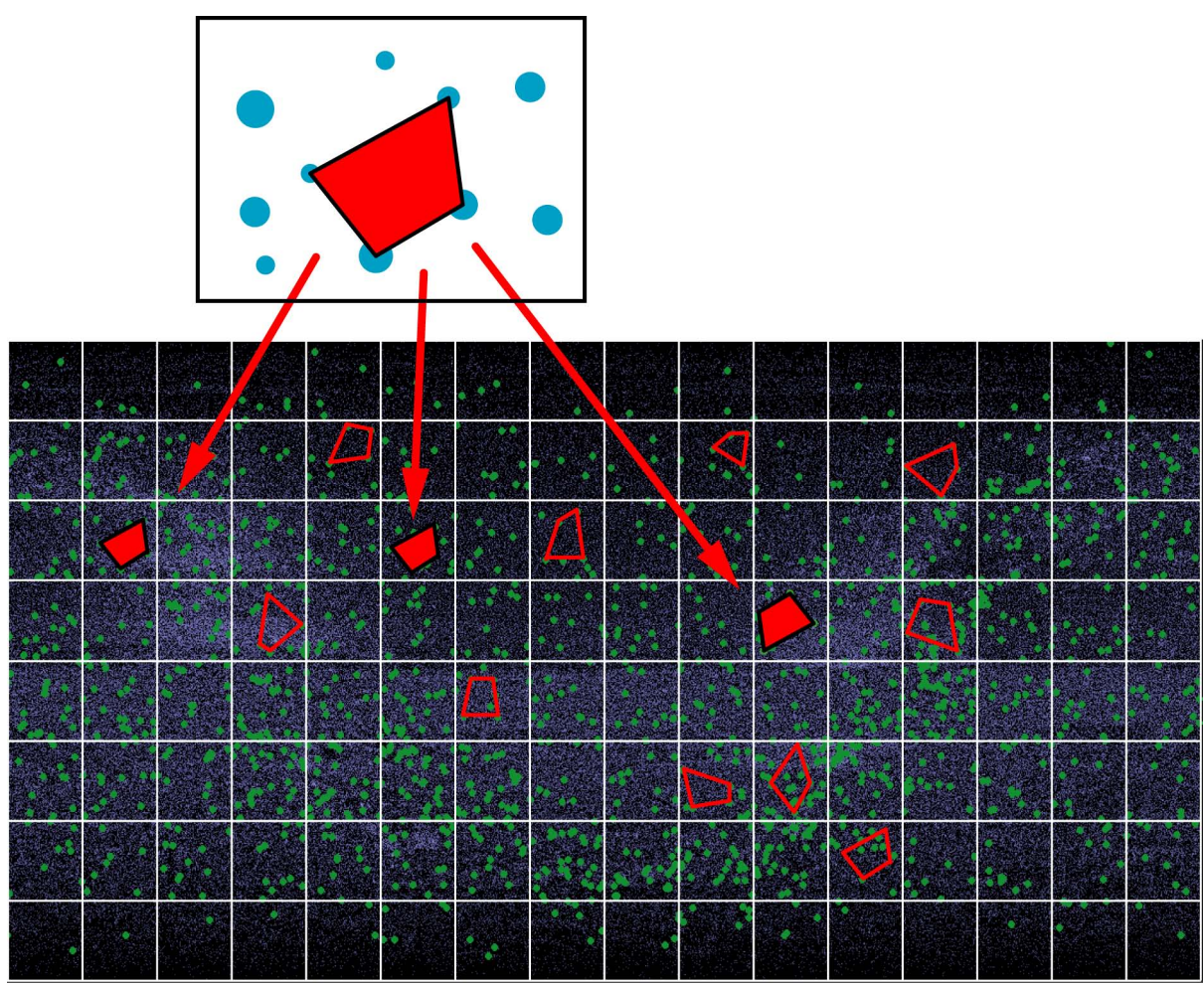

Fig. 3. Example of quads matching process

we would create convex quads in many different scales, whereas using a grid of seeds for the catalog would create only quads of similar sizes.

\section{Software}

The name 'Skyprint' 10] was intended to be analogous with fingerprinting because it tries to find the same patterns of stars in a large database. Skyprint is a highly interactive application written in $\mathrm{C}++$ with Qt interface. Sample screen of the program's graphical interface is shown in Fig. 5

There are two algorithms implemented in Skyprint. First, the algorithm presented in the previous section and second - a version of the algorithm presented by Valdes [1]. The distortion effects originating from projecting a celestial sphere onto a $2 D$ plane can no longer be neglected for photographs with FOV larger than 30 arcmin. For this reason Skyprint gives the option of choosing between three different types of projection. The first one is a 'no projection' method (the angles are calculated on the sphere with use of spherical trigonometry). The second method is to calculate the angles on the sphere by treating them as if they were in a two dimensional plane. The last one, the 'gnomonic projection', 


\begin{tabular}{l|l|l|l|l|l|l|l|l|l|l|l|l|l|l|l|l}
7 & 0 & 0 & 0 & 0 & 0 & 0 & 0 & 0 & 0 & 0 & 0 & 0 & 0 & 0 & 0 & 0 \\
\hline 6 & 0 & 0 & 0 & 0 & 0 & 0 & 0 & 0 & 0 & 0 & 0 & 0 & 0 & 0 & 0 & 0 \\
\hline 5 & 0 & & 0 & 0 & 0 & & 0 & 0 & 0 & 0 & 0 & 0 & 0 & 0 & 0 & 0 \\
\hline 4 & 0 & 0 & 0 & 0 & 0 & 0 & 0 & 0 & 0 & 0 & & 0 & & 0 & 0 & 0 \\
\hline 3 & 0 & 0 & 0 & 0 & 0 & 0 & 0 & 0 & 0 & 0 & 0 & 0 & 0 & 0 & 0 & 0 \\
\hline 2 & 0 & 0 & 0 & 0 & 0 & 0 & 0 & 0 & 0 & 0 & & 0 & 0 & 0 & 0 & 0 \\
\hline 1 & 0 & 0 & 0 & 0 & 0 & 0 & 0 & 0 & 0 & 0 & 0 & 0 & 0 & 0 & 0 & 0 \\
\hline 0 & 0 & 0 & 0 & 0 & 0 & 0 & 0 & 0 & 0 & 0 & 0 & 0 & 0 & 0 & 0 & 0 \\
\hline & 15 & 14 & 13 & 12 & 11 & 10 & 9 & 8 & 7 & 6 & 5 & 4 & 3 & 2 & 1 & 0
\end{tabular}

Fig. 4. Sample results of voting procedure

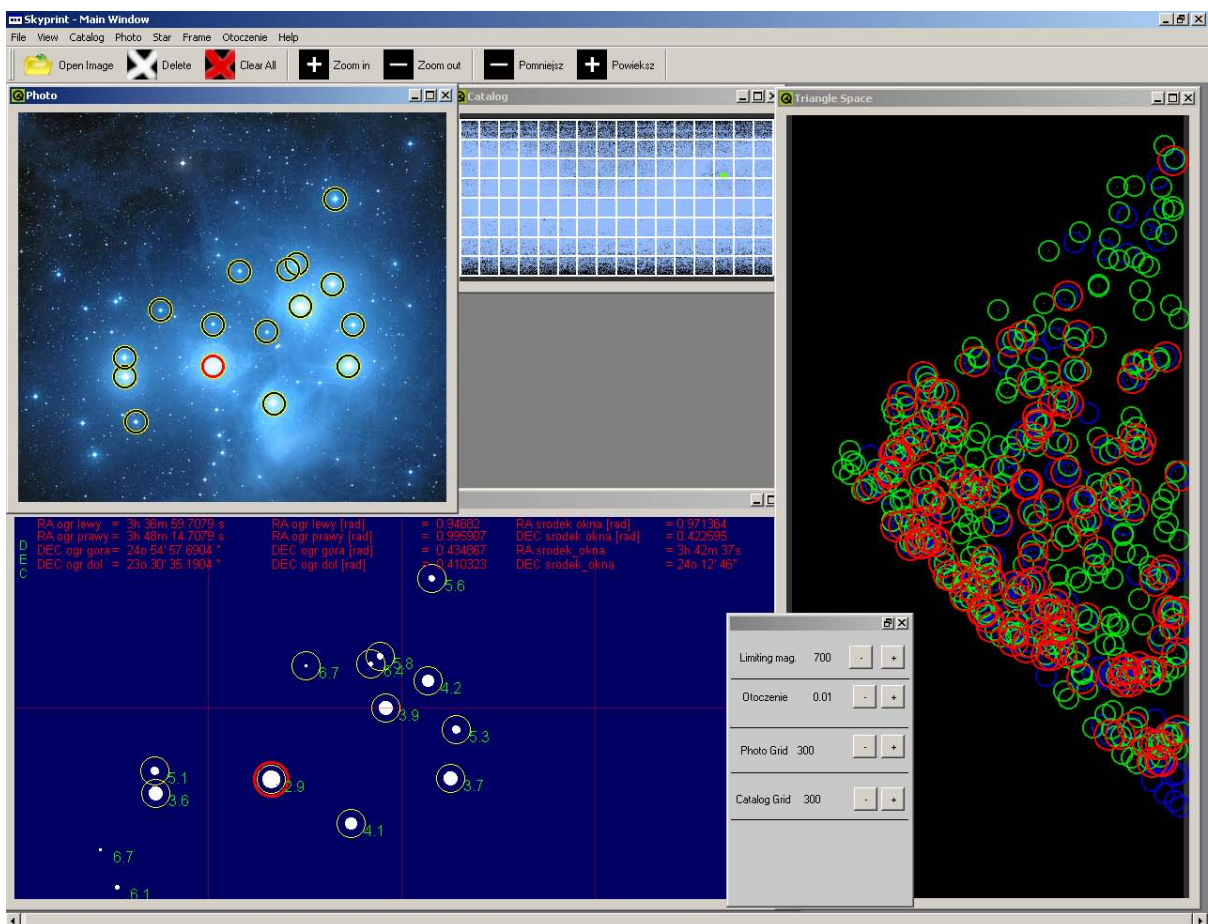

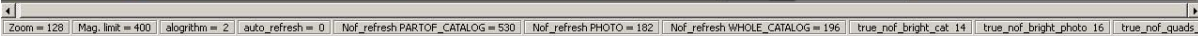

Fig. 5. Skyprint - view of the main window 
is the best option as it uses exactly the same distortion as the one that appears when a picture of the sky in taken.

The typical use case of Skyprint is as follows:

- Open the photograph.

- Select or correct position of stars on the photograph.

- Choose sky region (possibly the whole sky).

- Select between algorithm I and II.

- Choose matching parameters for the selected algorithm.

\section{Results}

Although we made our tests on a number of images, here we would like to concentrate on the comparison of results obtained by matching a single photograph while changing many parameters of the algorithm. We have used the simplest orthogonal division as shown in Fig. 3. This allowed us to divide the catalog into 128 parts. We placed seeds much more densely, but in precisely the same manner. Seed density is a parameter of the software. Typically we use two densities: $600 \times 300$ and 1200 x 600 . However, these seeds are not uniformly distributed on the sphere. This particular distribution causes the seeds with highest and lowest declinations to be close to other seeds. This is not desirable, because in this way there would be many more quads created for the regions close to the poles than for the parts of the sky which lie beside the equator. The same problem applies to choosing fragments that the catalog is divided into. One solution is to use

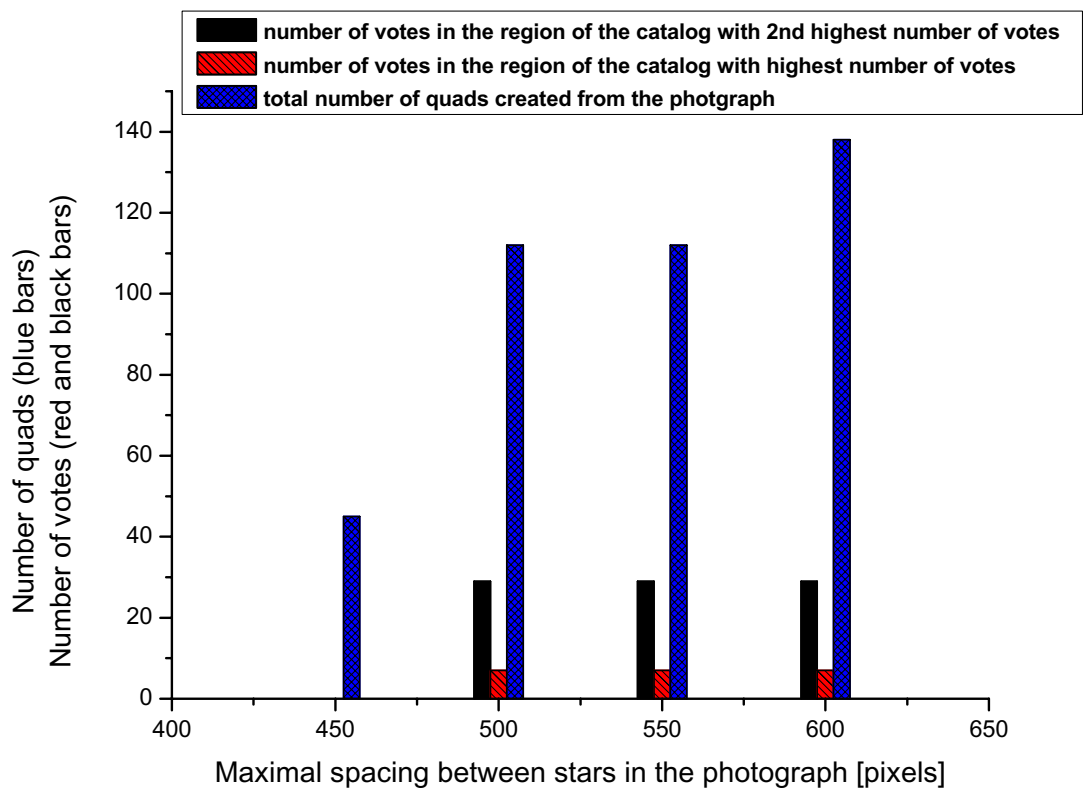

Fig. 6. Results obtained without using any projection 


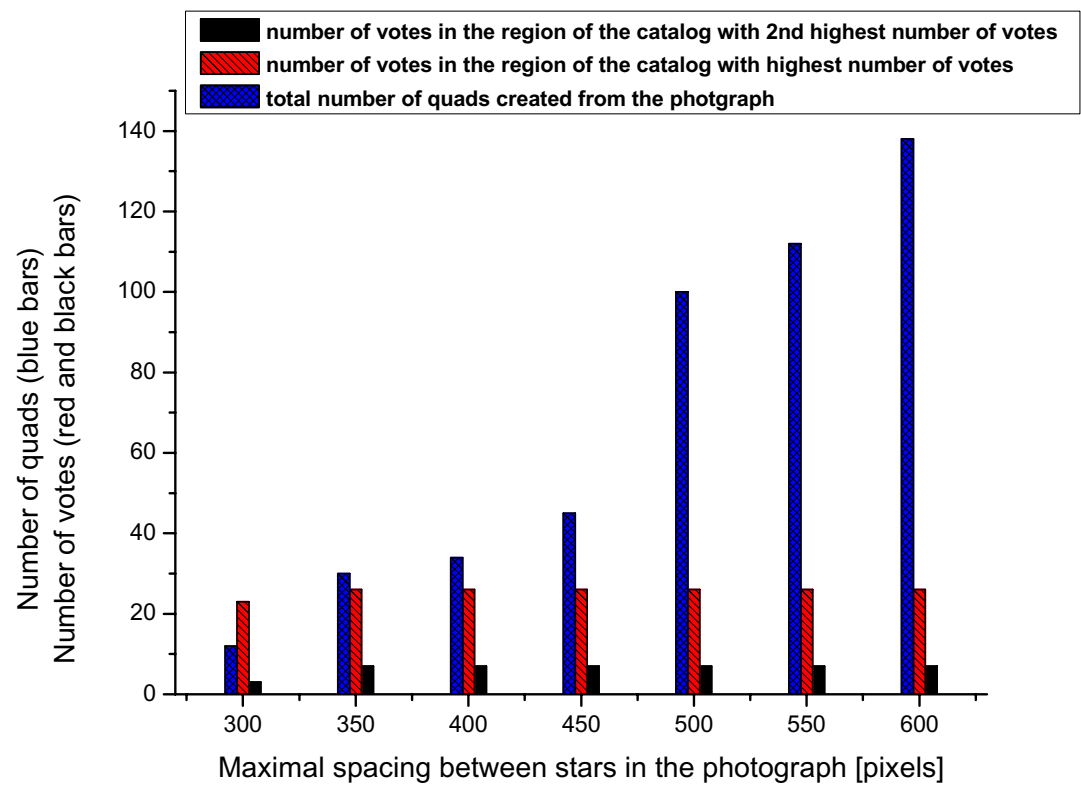

Fig. 7. Results obtained by calculating the angles on the sphere with use of spherical trigonometry

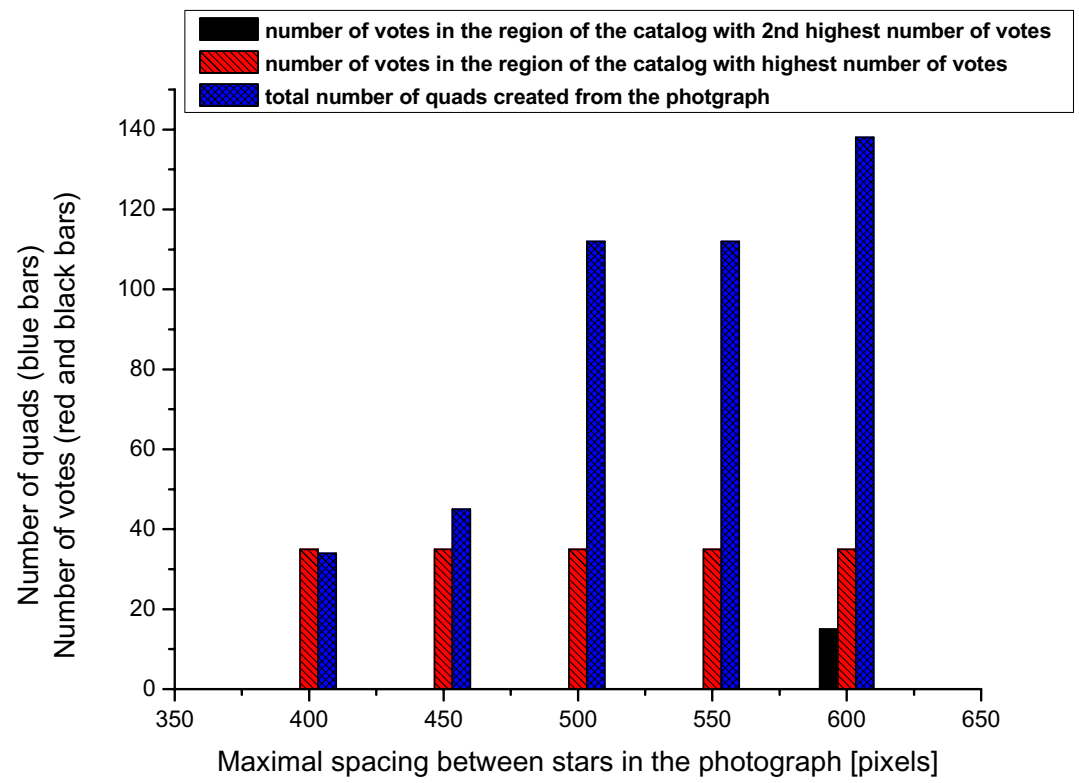

Fig. 8. Results obtained by using the gnomonic projection 
HEALPix [8] for determining the uniform distribution of seeds and regions of the catalog. The other is to utilize the constraint in equation 1 For the tests we used a M45 7] photograph with a FOV of about 1 degree, which is very small in size, when compared with 22.5 degrees (the largest part into which we divide the catalog). The larger the ratio of FOV to the size of the part of the catalog, the harder it is for the algorithm to work properly. This was one of the reasons for choosing this photograph. The second reason was to show that even at such a small FOV (small in the context of amateur photography) we need to make sure we pay attention to distortions. In Fig. 6, 7 and 8 we show the number of created quads compared with the number of votes in the regions with two highest ranks, obtained for each method of angle calculation.

\section{Conclusions}

We have shown that the implemented algorithm works well with almost any class of photographs. We have made no assumptions about the photograph apart from limiting its size which makes the algorithm much more efficient.

In practice the maximum size of the photograph is usually well known and can be set as a parameter, thus representing a very soft constraint.

We have also determined the most important factors in the identification of the fragment of the sky. One of them is the number of stars selected in the photograph either by an automatic filter or the user. The more stars selected, properly and precisely, the higher the probability of successful matching. The projection and the method used to calculate angles of the shape is also very important, especially for larger FOVs. For matching real life photographs of the sky, the gnomonic projection is the best choice. Because there are countless methods of characterizing shapes we plan to explore some more possibilities further in our future research on this subject.

The work of Arzoumanian et.al. 9, shows that different applications of the star pattern matching algorithms may exist in a disparate fields of science and are still to be explored.

AGH Grant no. 11.11.120.777 is acknowledged.

\section{References}

1. Valdes, F.G., Campusano, L.E., Velasquez, J.D., Stetson, P.B.: FOCAS Automatic Catalog Matching Algorithms. PASP 107, 1119 (1995)

2. Groth, E.J.: A pattern-matching algorithm for two-dimensional coordinate lists. The Astrophysical Journal 91, 1244-1248 (1986)

3. Murtagh, F.: A new approach to point-pattern matching. PASP 104, 301-307 (1992)

4. Lang, D., Hogg, D.W., Mierle, K., Blanton, M., Roweis, S.: Making the sky searchable (submitted, 2007), http://www . astrometry.net

5. Harvey, C.: New Algorithms for Automated Astrometry. M.Sc. Thesis, University of Toronto (2004) 
6. Roeser, S., Bastian, U.: PPM Star Catalog, vol. I \& II. Astronomisches RechenInstitut, Heidelberg (1991)

7. De Martin, D.: M45 picture, http://www.skyfactory.org

8. Górski, K.M., Hivon, E., Banday, A.J., Wandelt, B.D., Hansen, F.K., Reinecke, M., Bartelmann, M.: HEALPix: A Framework for High-Resolution Discretization and Fast Analysis of Data Distributed on the Sphere. The Astrophysical Journal 622(2), 759-771 (2005)

9. Arzoumanian, Z., Holmberg, J., Norman, B.: An astronomical pattern-matching algorithm for computer-aided identification of whale sharks Rhincodon typus. Journal of Applied Ecology - British Ecological Society 42 (6), 999-1011 (2005)

10. Makowiecki, W.: Skyprint software for interactive Star Pattern Matching, http://www.skyprint.info 